\title{
Plastic Deformation and Cavitation in Semi-crystalline Polymers
}

\author{
Andrzej Pawlak \\ Centre of Molecular and Macromolecular Studies, Polish Academy of Sciences, \\ Sienkiewicza 112, 90-363 Lodz, Poland
}

\begin{abstract}
The phenomenon of cavitation is observed in many semi-crystalline polymers during uniaxial stretching. The nano and micro size voids are formed inside the amorphous phase of polymer, usually beginning at yield. Cavitation happens when the strength of amorphous phase is lower than the strength of crystals. The process of plastic deformation and conditions at which polymers cavitate are discussed on the examples of polyethylene and polypropylene.
\end{abstract}

Keywords: plastic deformation, cavitation, polyethylene, polypropylene

PACS: $81.05 . \mathrm{Lg}, 81.40 . \mathrm{Lm}$, 81.70.Bt, 61.05.Cf, 07.85.Qe

\section{INTRODUCTION}

Cavitation phenomenon, meaning a formation of numerous voids (cavities) inside a solid material, is observed in many semi-crystalline polymers tensely deformed at temperatures above their glass transition. The semicrystalline polymers have a complex internal structure, are composed from crystalline elements-lamellae and amorphous phase [1]. Often in polymers are formed also a higher level structures- spherulites. The deformation of semi-crystalline polymer is a complicated, multistep process. It is widely accepted that existing spherulitic structure transforms into fibrillar. Although the plastic deformation of crystallizing polymers is studied for many years, still there are some aspects which are poorly recognized and understood [2]. One example is a formation of numerous cavities inside the amorphous phase on the beginning of crystals plastic deformation. It happens often, but not always in some polymers, under some circumstances. Observations confirm that cavities develop when the polymer is stretched, but do not form when the mode of deformation is shear or compression. It means that a local 3dimensional tensile stress is important for initiation of voiding process. Cavities formed in polymer are nano or micrometer size. Their presence may be detected by many methods. Most often used methods are: microscopies (SEM, TEM, AFM and polarized light microscopy), small angle X-ray scattering (SXAS) and measurements of density or volume change.

For many years the cavitation during deformation was treated as something existing, but do not really important for the mechanical properties of material. This opinion changed recently, when the new experimental facts were known [3-7]. Some of them were results obtained in CMMS laboratory. Our studies, done in a last few years, were focused on identification of the conditions of cavitation, characterization of the cavitation process and understanding how the formation of numerous voids affects the deformation in microscale and macroscopic behavior of plastics.

Usually the voids are detected close to yield or at yield, strongly suggesting that yielding is caused by cavitation. However, we explained [4] that there is a competition between two possible processes: breaking of the amorphous phase leading to cavitation and plastic deformation of the lamellar crystals. Which process occurs first depends on the relation between compliances of those two phases. If the crystals are weak and defected their deformation occurs at yield, mostly by chain slips mechanism and without cavitation in amorphous phase. This was observed, for example, for stretched LDPE. If the crystals in a polymer are thick and more perfect then the barrier for their deformation, represented by shear yielding stress, is increased and the cavitation sets in first and yielding is determined by the stress needed for cavitation. Further deformation involves deformation of crystals due to rapid local change of stress around voids [4,5]. 
The relation between strengths of amorphous and crystalline phase depends on the conditions for crystallization during solidification of polymer from melt state. Particularly sensitive for solidification procedure and especially for cooling rate is the crystalline structure. Our recent studies on cavitation were focused on understanding how the changes in crystalline structure influences the cavitation process. The polymers selected for studies were polypropylene and high density polyethylene, because their behavior are typical for the whole class of semicrystalline polymers. The experimental results are presented below.

\section{EXPERIMENTAL}

\section{Materials and Methods}

Polypropylene used in these studies was Malen P, F $401\left(\mathrm{M}_{\mathrm{w}} 297200 \mathrm{~g} / \mathrm{mol}, \mathrm{M}_{\mathrm{n}}=56400 \mathrm{~g} / \mathrm{mol}\right.$, MFR 3g/10 min (at $\left.190{ }^{\circ} \mathrm{C}, 2.16 \mathrm{~kg}\right)$ ), produced by Basell Orlen Polyolefins. The second polymer, high density polyethylene (HDPE) Lupolene $6021 \mathrm{D}\left(\mathrm{M}_{\mathrm{w}}=1.8 * 105 \mathrm{~g} / \mathrm{mol}, \mathrm{M}_{\mathrm{w}} / \mathrm{M}_{\mathrm{n}}=7.2\right)$, was produced by BASF.

Samples for the mechanical test were formed both by injection molding and compression molding. In the second case a dog-bone shape specimens were cut from 0.5-1 mm thick films. Several cooling procedures were applied in aim to achieve different crystallinity and crystalline structure of plastics. The molten samples were cooled in iced water, water or on the air. The last procedure gave longer time for crystallization and resulted in highest crystallinity and crystal thickness. Some samples were crystallized isothermally by using a laboratory heating device. Crystallization process and crystallinity of materials were characterized by differential scanning calorimetry (DSC). Mechanical properties of polymers were studied in uniaxial drawing, using „Instron 5582” tensile machine. The rate of deformation was $5 \% / \mathrm{min}$ (i.e. $8.3 * 10^{-4} \mathrm{~s}^{-1}$ ). During the test were also determined a local strain and volume increase by video measurements of momentary dimensions of the sample in three main directions.

Because the best way of characterization of nano voids is a small angle X-ray scattering (SAXS), many such experiments were done in our laboratory. SAXS patterns were used for detection of cavities (with dimensions in range of 2-80 nm) and for determination of parameters of crystalline structure, i.e. long period and crystal thickness. Some SAXS measurements were performed using a power full synchrotron radiation source in Hasylab laboratory (Hamburg). The short time of acquisition gave possibilities for registration of patterns in situ during deformation of samples. This deformation was done by using a laboratory tensile machine. The technical characteristic of this device was similar to "Instron", with exception of force limit of $100 \mathrm{~N}$. WAXS technique was used for studies of lamellar structure evolution and reorganization. The spherulitic morphology of plastics was examined by using a polarizing light microscope and a "Jeol" scanning electron microscope.

\section{Results and discussion}

Figure 1 presents the stress-strain dependence and small angle X-ray scattering patterns registered for uniaxialy deformed high density polyethylene samples. The material was prepared by crystallization from melt, however with two different cooling procedures: quick cooling in the iced water (Fig. 1a) and slow cooling in the air (Fig. 1b). Both analyzed samples deformed plastically with a formation of neck. The quickly cooled polymer has yield stress of 21 $\mathrm{MPa}$, the second one has higher yield level of $29 \mathrm{MPa}$. Figure 1 also presents SAXS patterns, measured at selected deformations. The formation of nano size cavities is visible on these patterns as a rapid increase of scattering intensity and clearly it happened for HDPE sample previously cooled in air. The voids were not formed in this polyethylene specimen, which was crystallized with fast cooling (Fig 1a). The cavities were observed first time around the yield point. The shapes of patterns suggest that voids in PE were ellipsoidal and initially were elongated perpendicularly to the deformation direction. The reorientation of voids into deformation direction happened for strain of $50 \%$. Such reorientation was result of transformation in crystalline phase surrounding voids, which was confirmed by WAXS observations.

Some polymers have several allotropic modifications of the crystalline structure. An example is isotactic polypropylene which may crystallize in three different forms: monoclinic $\alpha$, trigonal $\beta$, and triclinic $\gamma$. The most common and the most stable is the $\alpha$ form, characterized by the so-called "cross-hatched" spherulitic morphology, in which radial and tangential lamella coexist. The crystals of $\beta$ form grow faster than those of $\alpha$ form and spherulites of $\beta$ PP exhibit exclusively radial lamellar architecture. The third possible form, triclinic $\gamma$, occurs only in specific crystallization conditions. The polymers of $\alpha$ and $\beta$ crystallographic forms exhibit similarity of behavior in the tensile test. It was expected that differences between crystallographic forms should modify the cavitational behavior 
of polypropylene. Two polypropylene specimens prepared for mechanical studies contained respectively: $99.9 \%$ of $\alpha$ form crystals ( $\mathrm{PP} \alpha)$ and $90 \%$ of $\beta$ form crystals $(\mathrm{Pp} \beta)$. The rest of crystalline phase for second sample was $\alpha$ form.

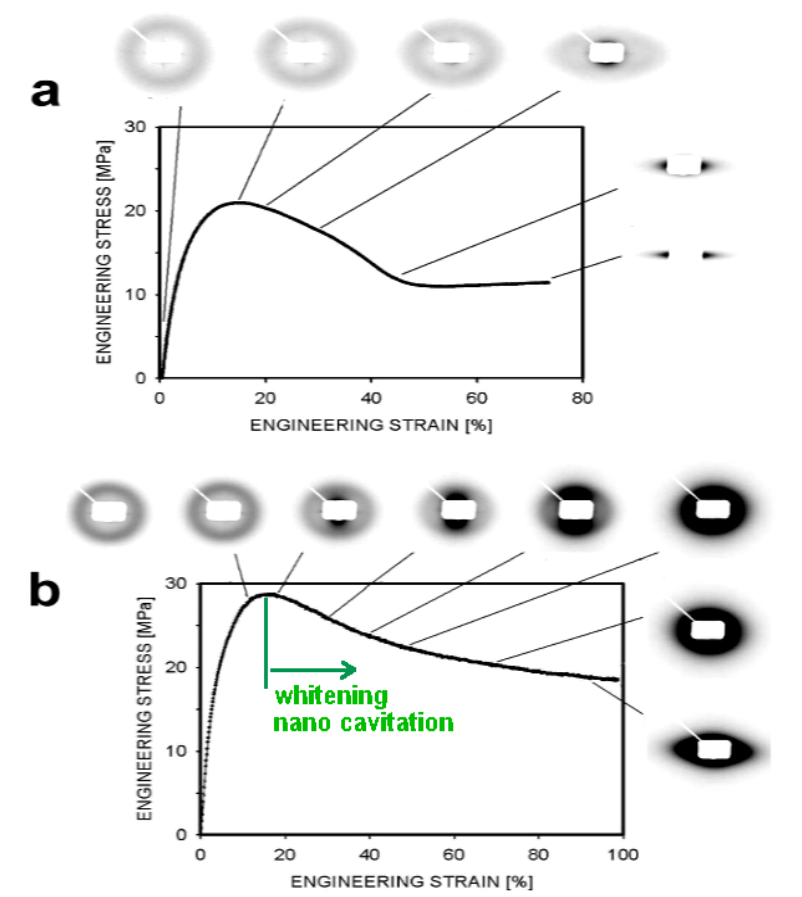

FIGURE 1. Stress-strain dependences and SAXS patterns registered during tensile deformation of high density polyethylene samples prepared with different cooling procedures: a) rapid cooling in iced water, b) slow cooling in the air. Deformation direction on patterns is vertical.

The mechanical behavior of drawn polypropylene (PP) samples and related SAXS patterns are presented in Figure 2. It is visible the decrease of yield stress in $\beta$ phase rich sample. The whitening of samples, indicating the presence of micrometer size voids, happened near the yield point. The generation of nano voids in deformed samples was confirmed by changes of intensities in X-ray scattering patterns, shown also in Fig. 2. These nano cavities were visible since yield point. The intensity of scattering increased with deformation, which means that more voids was generated and that those existing enlarged. The evolution of cavities with time of deformation was similar to this observed for polyethylene. The most important conclusion from SAXS test of polypropylene was that the scale of cavitation was larger when the polymer contained more crystals of $\beta$ form.

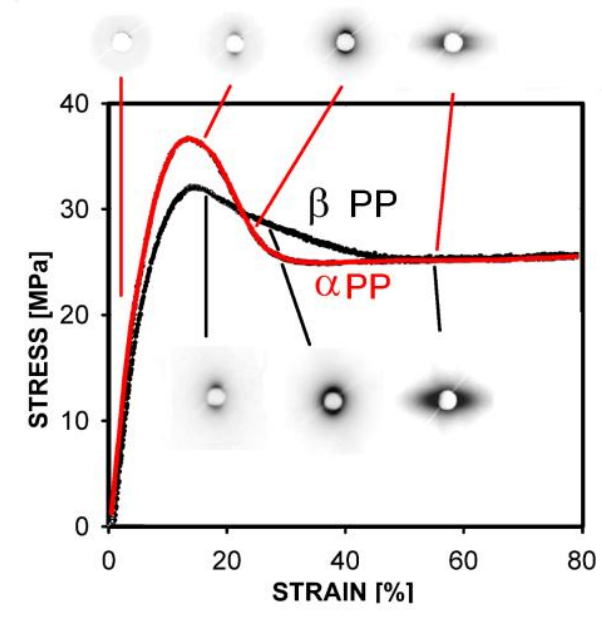

FIGURE 2. Stress-strain dependences for PP specimens containing $99.9 \%$ of $\alpha$ phase crystals (red curve) and $90 \%$ of $\beta$ phase crystals (black curve). SAXS patterns are also shown for selected strains. Strain direction on these patterns was vertical. 
The analysis of scattering profile allowed for determination of the gyration radius $\mathrm{R}$, which is a measure of voids' size. The conditions for applying Guinier's approach were accomplished at the beginning of plastic deformation, therefore the gyration radii were determined only for samples strained to $20 \%$. In each PP sample two populations of voids were found, characterized by the gyration radii of 5 and $13 \mathrm{~nm}$.

The measurements of a local volume in most deformed parts of each sample showed that this volume increased with deformation for both forms of polypropylene. Initially, to the strain of $10-15 \%$, the increase weakly depended on the $\beta$ phase content. For larger strains the differences between samples were visible. The volume of PP $\alpha$ sample increased with deformation to the engineering strain of $40 \%$, when it reached value of 1.35 , but later this volume remained approximately constant. The increase of volume was very fast for PP $\beta$ specimen. At the strain of $25 \%$ this volume was 1.65 . The volume increased linearly for large strains. The largest value of volume $\mathrm{V} / \mathrm{Vo}=2.9$ was measured for PP $\beta$ sample deformed to the strain of $80 \%$. In the semi-crystalline polymers nearly all of the volume increase is attributed to the formation of cavities. The larger scale of cavitation in $\beta$ spherulites of polypropylene results from differences in their structure. Spherulites of $\beta$ form, have only a radial arrangement of lamellae, without tangential lamellae present in $\alpha$ spherulites and this support the cavitation process.

\section{CONCLUSIONS}

The cavitation is observed in many semi-crystalline polymers deformed in tension, but never in compression. It was shown on the examples of polypropylene and polyethylene that if the crystallization process is rapid and a lot of defects is generated in crystals then polymer does not cavitate during tensile deformation. More slow crystallization during solidification results in cavitation, first time visible at yield. In cavitating polymer on the beginning of plastic deformation dominates nano size voids. Morphological factors may support intensification of cavitation. The example is $\beta$-form polypropylene where radial architecture of spherulites lead to more intensive cavitation, when compared with cavitation in "cross-hatched" structure of $\alpha$-form spherulites. Other example is polyethylene crystallized in isothermal conditions.

\section{ACKNOWLEDGMENTS}

I wish to express thanks to the Hamburg Synchrotron Radiation Laboratory for the beam time granted within the project I 20100253 EC. The project was financed from funds of the National Science Centre on the basis of the decisions number DEC-2011/03/D/ST8/04156 and 2012/04/A/ST5/00606.

\section{REFERENCES}

1. P. B. Bowden and R. J. Young, J. Mater. Sci., 9, 2034-2951 (1974).

2. A. Galeski, Prog. Polym. Sci., 28, 1643-1699, (2003).

3. A. Pawlak and A. Galeski, Macromol., 38, 9688-9697 (2005).

4. A. Pawlak and A. Galeski, J. Polym. Sci., Part B: Pol. Phys., 48, 1271-1280 (2010).

5. S. Humbert, O. Lame, J. M. Chenal, C. Rochas, G. Vigier, Macromol., 43, 7212-7221 (2010).

6. A. Pawlak, Coll. Polym. Sci., 291, 773-787 (2013).

7. L. Farge, S. Andre, A. Pawlak, Ch. Baravian, S.C. Irvine, A.M. Philippe, J. Polym. Sci., Polym. Phys., 51, 826-841, (2013). 